\title{
ANALYTIC SYSTEMS OF CENTRAL CONICS IN SPACE
}

\author{
BY \\ J. L. COOLIDGE
}

The amount of literature dealing with conic sections, individual curves and systems of curves, in one plane, is vast. When however we are dealing with a number of conics, not in the same plane, the situation is quite different. Certain figures, as the focal conics of a set of confocal quadrics, are familiar enough, but very little has been done in the way of a systematic study of more general systems. There are some studies carried out with the aid of purely synthetic methods; the algebraic or analytic treatment lags behind.

The first writer to suggest a reasonable set of coordinates for a conic in space was Spottiswoode $\left({ }^{1}\right)$. The totality of straight lines that intersect a conic in three-space generates a very special sort of quadratic complex. The coefficients determining the equation of this complex, when a straight line has the usual Plücker line coordinates, may be taken as the coordinates of the conic, a clumsy enough system. A much better technique, perhaps the best for algebraic purposes, was developed by Johnson $\left({ }^{2}\right)$. Here a conic is looked upon not as a locus, but as the envelop of its tangent planes. Thus its tangential equation $a^{i j} u_{i} u_{j}=0$ gives ten homogeneous coordinates, connected by a quartic identity

$$
a^{i j}=a^{j i}, \quad\left|a^{i j}\right|=0, \quad i, j=1,2,3,4 .
$$

I think that this gives the best approach to the study of algebraic systems of conics, and I regret that more attention has not been given to the subject. For instance a complete study of linear and quadratic systems would be interesting. When it comes to attacking differential properties of conics this technique is disappointing, even as the Plü ker line coordinates are of comparatively little use in studying the differential properties of systems of lines. In fact as far as I can make out very little has been written about the differential geometry of systems of conics. The most important article I have been able to find was by Blutel $\left({ }^{3}\right)$, and his problem is very special. In what follows I am going to outline what seems to me the most promising way to approach the subject, and give a certain number of theorems. I hope that others may feel inclined to carry the study further, even though present mathematical fashion is concerned with very different questions.

Presented to the Society, April 27, 1940; received by the editors December 1, 1939.

(1) On the twenty-one coordinates of a conic in space, Transactions of the London Mathematical Society, vol. 10 (1879).

(2) The conic as a space element, these Transactions, vol. 15 (1914).

(3) Recherches sur les surfaces qui sont en même temps lieux de coniques et enveloppes de cones, Annales de l'École Normale Supérieure, (3), vol. 7 (1890). 
1. Series of conics. The most obvious way to approach the study of the conic in space is to treat it as a rational curve. Let us use nonhomogeneous Cartesian coordinates, and assume that our conic is not a parabola. We may then write its parametric equations in the form

$$
x^{i}=a^{i} t+b^{i}+c^{i} \frac{1}{t}, \quad i=1,2,3 .
$$

Here, $(a)$ and $(c)$ give the directions of the asymptotes, $(b)$ are the coordinates of the centre and, if we assume that $t=1$ gives a vertex, $t^{2}$ is the ratio of the distances from the asymptotes.

I give these equations because they seem to offer a favorable opening for the study of systems of conics, and in fact I personally first tried the problem in this way. I hasten to add that I was not at all able to attain the results which I believe to be easily attainable.

I turn to a different method which seems to fit the case even better. This is the method of moving axes first developed by Darboux in the opening chapters of his Théorie Générale des Surfaces, and extended in recent years by Cartan. Let a point have the rectangular Cartesian coordinates $\left(X^{i}\right)$ with regard to a set of fixed axes. Its coordinates with respect to a moving set of such axes shall be $\left(x^{i}\right)$. The coordinates with regard to the fixed axes of the moving origin shall be $\left(X_{0}^{i}\right)$. We then have the fundamental relations

$$
X^{i}=X_{0}^{i}+a_{i j} x^{j}, \quad a_{i j}=\frac{\partial\left|a_{p q}\right|}{\partial a_{i j}} ; \quad\left|a_{p q}\right|=1 .
$$

Let the position of the point and also the situation of the moving axes be functions of a parameter $v$, which for simplicity of language I shall call "time." We then have

$$
\frac{\partial X^{i}}{\partial v}=\frac{\partial X_{0}^{i}}{\partial v}+\frac{\partial a_{i j}}{\partial v} x^{j}+a_{i j} \frac{\delta x^{j}}{\delta v} .
$$

I now seek the components with regard to the moving axes of the total velocity of the point. We write these $\partial x^{i} / \partial v$, while we mean by the notation $\delta x^{i} / \delta v$ the velocity with regard to the moving axes of the point's motion with regard to those same axes:

$$
\begin{gathered}
\frac{\partial x^{i}}{\partial v}=\xi^{i}+\frac{\delta x^{i}}{\delta v}+q_{i j} x^{j} \\
q_{i j}=-q_{j i}=a_{k i} \frac{\partial a_{k j}}{\partial v}, \quad \xi^{i}=a_{k i} \frac{\partial X_{0}^{k}}{\partial v} .
\end{gathered}
$$

It is to be remembered that $\left\|a_{i j}\right\|$ is the matrix of an orthogonal substitution of determinant 1. 
These are the general formulae for moving rectangular axes in any number of dimensions. For our particular problem, let us assume that our conic lies in the plane $x^{3}=0$ and that it is expressed parametrically

$$
x^{1}=a \cos u, \quad x^{2}=b \sin u, \quad x^{3}=0 .
$$

Let us further simplify the notation by writing $i, j, k$ as a cyclic permutation of $1,2,3$ and putting

$$
q_{i j}=-p_{k} .
$$

We have then our fundamental formulae

$$
\begin{aligned}
\frac{\partial x^{1}}{\partial u}=-a \sin u, & \frac{\partial x^{1}}{\partial v}=\xi^{1}+\frac{\partial a}{\partial v} \cos u-p_{3} b \sin u, \\
\frac{\partial x^{2}}{\partial u}=b \cos u, & \frac{\partial x^{2}}{\partial v}=\xi^{2}+\frac{\partial b}{\partial v} \sin u+p_{3} a \cos u, \\
\frac{\partial x^{3}}{\partial u}=0, & \frac{\partial x^{3}}{\partial v}=\xi^{3}+p_{1} b \sin u-p_{2} a \cos u .
\end{aligned}
$$

We have further in the classical notation

(8)

$$
\begin{aligned}
& E=a^{2} \sin ^{2} u+b^{2} \cos ^{2} u \\
& F=b \xi^{2} \cos u-a \xi^{1} \sin u+\frac{1}{2} \frac{\partial\left(b^{2}-a^{2}\right)}{\partial v} \cos u \sin u+p_{3} a b \\
& G=\sum_{i}\left(\xi^{2}\right)^{2}+2\left[\xi^{1} \frac{\partial a}{\partial v}+\left(\xi^{2} p_{3}-\xi^{3} p_{2}\right) a\right] \cos u \\
&+2\left[\xi^{2} \frac{\partial b}{\partial v}+\left(\xi^{3} p_{1}-\xi^{1} p_{3}\right) b\right] \sin u \\
&+\left[\left(p_{2}^{2}+p_{3}^{2}\right) a^{2}+\left(\frac{\partial a}{\partial v}\right)^{2}\right] \cos ^{2} u \\
&-2\left[p_{3}\left(b \frac{\partial a}{\partial v}-a \frac{\partial b}{\partial v}\right)^{2}+p_{1} p_{2} a b\right] \cos u \sin u \\
&+\left[\left(p_{3}^{2}+p_{1}^{2}\right) b^{2}+\left(\frac{\partial b}{\partial v}\right)^{2}\right] \sin ^{2} u
\end{aligned}
$$

$$
\begin{aligned}
\frac{\partial\left(x^{1}, x^{2}\right)}{\partial(u, v)}=- & {\left[a \xi^{2} \sin u+b \xi^{1} \cos u+b \frac{\partial a}{\partial v} \cos ^{2} u\right.} \\
& \left.-p_{3}\left(b^{2}-a^{2}\right) \cos u \sin u+a \frac{\partial b}{\partial v} \sin ^{2} u\right]
\end{aligned}
$$




$$
\begin{aligned}
\left(E G-F^{2}\right)^{1 / 2} D= & -a b \frac{\partial x^{3}}{\partial v} \\
\left(E G-F^{2}\right)^{1 / 2} D^{\prime}= & \frac{\partial^{2} x^{3}}{\partial u \partial v} \frac{\partial\left(x^{1}, x^{2}\right)}{\partial(u, v)}-\frac{\partial x^{3}}{\partial v}\left[\left(b \frac{\partial a}{\partial v}-a \frac{\partial b}{\partial v}\right) \cos u \sin u\right. \\
& \left.+p_{3}\left(b^{2} \cos ^{2} u+a^{2} \sin ^{2} u\right)\right] .
\end{aligned}
$$

If we have given two conics, they may have any one of the five following relations: (a) they do not intersect, (b) they intersect once, (c) they intersect twice, (d) they touch, and (e) they may be coplanar. Omitting the last case, when we are considering a one-parameter family of conics in space, we have to distinguish the cases where adjacent conics do not meet, or where they meet once, or where they meet twice, or where they are tangent to one another. Or to put the matter in more exact language, they may not all touch any curve, or they may all touch a curve, or they may touch two curves (or one curve twice), or they may all touch a curve and lie in the corresponding osculating planes. Let us verify these statements analytically.

If the conics of a series touch one curve, it must be possible to make $u$ such a function of $v$ that

$$
\frac{\partial x^{i}}{\partial u}=\rho \frac{\partial x^{i}}{\partial v}
$$

This involves

$$
\frac{\partial x^{3}}{\partial v}=0, \quad \frac{\partial\left(x^{1}, x^{2}\right)}{\partial(u, v)}=0 .
$$

If we replace the sine and cosine of $u$ by $(2 t) /\left(1+t^{2}\right),\left(1-t^{2}\right) /\left(1+t^{2}\right)$, where $t$ is the tangent of half of the excentric angle, we have the condition that the resultant of a quadratic and a quartic polynomial in $t$ should vanish, which is a bit long to write out, but involves no theoretical difficulties. There is more interest in the case where the conics touch two curves.

These curves lie on the developable surface generated by the plane of the conic, the characteristic line being the intersection with

$$
\frac{\partial x^{3}}{\partial v} \equiv \xi_{3}+p_{1} b \sin u-p_{2} a \cos u=0 .
$$

The points where $\partial\left(x^{1}, x^{2}\right) / \partial(u, v)=0$ must include the two intersections of the conic with $\partial x^{3} / \partial v=0$ so that

$$
\frac{\partial\left(x^{1}, x^{2}\right)}{\partial(u, v)} \equiv \frac{\partial x^{3}}{\partial v}(\alpha \cos u+\beta \sin u+\gamma) .
$$


Again look at the matter geometrically. When two conics intersect twice, they lie on a pencil of quadric surfaces, two of which are cones, and the vertices of these cones are harmonically separated by the planes of the conics. When the conics are infinitely near, one cone tends to be squashed between them. It appears then that if the conics of our series are twice tangent to a curve, the tangent planes to the surface generated at all points of a conic pass through a common point and envelop a cone. Now let us look at the matter analytically. The equation of the tangent plane is

$$
\begin{array}{r}
\left(X^{1}-x^{1}\right) \frac{\partial\left(x^{2}, x^{3}\right)}{\partial(u, v)}+\left(X^{2}-x^{2}\right) \frac{\partial\left(x^{3}, x^{1}\right)}{\partial(u, v)}+\left(X^{3}-x^{3}\right) \frac{\partial\left(x^{1}, x^{2}\right)}{\partial(u, v)}=0, \\
{\left[X^{1}(b \cos u)+X^{2}(a \sin u)-a b\right] \frac{\partial x^{3}}{\partial v}+X^{3} \frac{\partial\left(x^{1}, x^{2}\right)}{\partial(u, v)}=0 .}
\end{array}
$$

The reader should not confuse $X$ appearing here with that in (1). This becomes in the present instance, thanks to (11),

$$
X^{1} b \cos u+X^{2} a \sin u+X^{3}[\alpha \cos u+\beta \sin u+\gamma]-a b=0 .
$$

It appears then that the point

$$
X^{1}=-a \alpha / \gamma, X^{2}=-b \beta / \gamma, X^{3}=a b / \gamma
$$

is in the tangent plane at every point of the conic. Conversely, when these tangent planes pass through such a point, we have an identity in $v$ and $\partial\left(x^{1}, x^{2}\right) / \partial(u, v)$ is divisible by $\partial x^{3} / \partial v$ so that the conics touch two curves or are the limits of conics touching two curves.

THEOREM 1. If the conics of a series are tangent to two curves, the tangent planes to the surface generated at all points of a conic will envelop a quadric cone which touches the surface all along the conic.

Now consider the dual. We have a one-parameter family of quadric cones. If adjacent cones tend to touch twice, that is to say, if the cones are inscribed in two developable surfaces, they will also intersect in a conic.

THEOREM 2. If the quadric cones of a one-parameter series be inscribed in two different developables, the characteristic curves of these cones will be the generators of these developables and a series of conics tangent to two curves, or the limit of such a series.

The surfaces generated by these conics are the ones considered by Blutel (q. v.).

There remains the case where adjacent conics tend to touch. This means that $\partial\left(x^{1}, x^{2}\right) / \partial(u, v)$ is divisible by $\partial x^{3} / \partial v$ but the line

$$
\frac{\partial x^{3}}{\partial v}=0
$$


is tangent to the conic. If $P$ be the point of contact, its line of advance is along the conic, and also along the characteristic line whose equation has just been written. Hence $P$ must be the point of contact with the edge of regression. The plane of the conic must then be the osculating plane for the curve generated by $P$. Hence we have a series of conics tangent to a curve each lying in the corresponding osculating plane. Here also there will be a quadric cone tangent at all points of the conic.

We have assumed (11) here, that is,

$$
-\quad \frac{\partial\left(x^{1}, x^{2}\right)}{\partial(u, v)} \equiv \frac{\partial x^{3}}{\partial v}(\alpha \cos u+\beta \sin u+\gamma) .
$$

This identity will lead to the equations

$$
\begin{gathered}
-p_{2} a \alpha+\gamma \xi^{3}=-b \frac{\partial a}{\partial v}, \quad p_{1} b \beta+\gamma \xi^{3}=-a \frac{\partial b}{\partial v} \\
p_{1} b \alpha-p_{2} a \beta=p_{3}\left(a^{2}-b^{2}\right), \\
\alpha \xi^{3}-\gamma a p_{2}=-b \xi^{1}, \quad \beta \xi^{3}+\gamma b p_{1}=-a \xi^{2} .
\end{gathered}
$$

We see geometrically that if two conics lie on the same quadric cone, the generators of the cone establish a projective relation between them. When the conics are infinitely near, the generators give the directions of the curves conjugate to the conics in the surface generated. Analytically, the differential equation for the curves conjugate to the conics $\delta v=0$ is

$$
\begin{gathered}
D d u+D^{\prime} d v=0 \\
-a b \frac{\partial x^{3}}{\partial v} d u+\left[\frac{\partial^{2} x^{3}}{\partial u \partial v} \frac{\partial\left(x^{1}, x^{2}\right)}{\partial(u, v)}-\frac{\partial x^{3}}{\partial v}\left\{\left(b \frac{\partial a}{\partial v}-a \frac{\partial b}{\partial v}\right) \cos u \sin u\right.\right. \\
\quad+p_{3}\left(b^{2} \cos ^{2} u+a^{2} \sin ^{2} u\right) d v=0 .
\end{gathered}
$$

In the present case this is

$$
\begin{aligned}
-a b d u+ & {\left[\left(p_{1} b \cos u+p_{2} a \sin u\right)(\alpha \cos u+\beta \sin u+\gamma)\right.} \\
& -\left\{\left(b \frac{\partial a}{\partial v}-a \frac{\partial b}{\partial v}\right) \cos u \sin u\right. \\
& \left.\left.+p_{3}\left(b^{2} \cos ^{2} u+a^{2} \sin ^{2} u\right)\right\}\right] d v=0 .
\end{aligned}
$$

In view of (12) this becomes

$$
d u+[L(v)+M(v) \cos u+N(v) \sin u] d v=0 .
$$

Let us now introduce the tangent of the half-angle, so that 


$$
\begin{gathered}
\cos u=\frac{1-t^{2}}{1+t^{2}}, \quad \sin u=\frac{2 t}{1+t^{2}}, \quad d u=\frac{d t}{1+t^{2}}, \\
\frac{d t}{d v}+A(v)+B(v) t+C(v) t^{2}=0 .
\end{gathered}
$$

This is a Riccati equation, characterized by the fact that the cross ratio of four solutions is constant. This gives

Blutel's Theorem 3. If the central conics of a series be not coplanar, but touch two curves, the conjugate curves on the surface they generate will establish a projective correspondence among them $\left(^{4}\right)$.

Let us now look at the orthogonal trajectories of conics. Their differential equation is

$$
E d u+F d v=0 .
$$

Introducing the tangent of the half-angle as before, we have

$$
\begin{aligned}
2\left[b^{2} t^{4}+\right. & \left.\left(4 a^{2}-2 b^{2}\right) t^{2}+b^{2}\right] d t+\left[b \xi^{2}\left(1-t^{4}\right)\right. \\
& \left.-2 a \xi^{1} t\left(1+t^{2}\right)+\frac{\partial\left(b^{2}-a^{2}\right)}{\partial v} t\left(1-t^{2}\right)+p_{3} a b\left(1+t^{2}\right)^{2}\right]\left(1+t^{2}\right) d v=0 .
\end{aligned}
$$

These trajectories will establish a projective correspondence if this is a Riccati equation. If $b^{2}=a^{2}$, the equation reduces automatically to the Riccati form. Suppose that $b^{2} \neq a^{2}$. Then $1+t^{2}$ cannot divide the coefficient of $d t$ and the first factor in the coefficient of $d v$ must be proportional to the coefficient of $d t$. Evidently, the factor of proportionality must be zero, so that $F=0$ or

$$
\xi^{1}=0, \quad \xi^{2}=0, \quad p_{3}=0, \quad b^{2}-a^{2}=k,
$$

where $k$ is a constant, not zero.

THEOREM 4. The necessary and sufficient condition that the trajectories orthogonal to the central conics of a series should establish a projective correspondence among them is that the conics should be circles; or the centre should be fixed or move orthogonally to the plane, the distance between the foci should be constant. and the axes should not twist.

Let us now try to discover under what circumstances these orthogonal trajectories are geodesic curves of the surface. The necessary and sufficient condition for this is that

$$
\frac{\partial}{\partial u}\left(\frac{E G-F^{2}}{E}\right)=0
$$

(4) Blutel, loc. cit., p. 155. 
This shows that $F^{2}$, and so $F$, is divisible by $E$ when the roots of $E$ are distinct, and as they are of the same order in $t$ when we substitute the tangent of the half-angle, the factor must be a function of $v$ :

$$
F \equiv f(v) E,
$$

$f(v)\left[a^{2} \sin ^{2} u+b^{2} \cos ^{2} u\right]$

$$
\equiv\left[b \xi^{2} \cos u-a \xi^{1} \sin u+\frac{1}{2} \frac{\partial\left(b^{2}-a^{2}\right)}{\partial v} \cos u \sin u+p_{3} a b\right] .
$$

It follows from this identity that $f(v)=0, F=0$ or $\xi^{1}=\xi^{2}=p_{3}=0, b^{2}-a^{2}=c$, where $c$ is a constant, not zero.

Thus $\partial G / \partial u=0$, or

$\xi^{3} p_{1} b \cos u+\xi^{3} p_{2} a \sin u+p_{1} p_{2} a b\left(\sin ^{2} u-\cos ^{2} u\right)$

$$
+\left[p_{1}^{2} b^{2}+\left(\frac{\partial b}{\partial v}\right)^{2}-p_{2}^{2} a^{2}-\left(\frac{\partial a}{\partial v}\right)^{2}\right] \sin u \cos u \equiv 0 .
$$

Hence

$$
\xi^{3} p_{1}=\xi^{3} p_{2}=p_{1} p_{2}=p_{1}^{2} b^{2}-p_{2}^{2} a^{2}+\left(\frac{\partial b}{\partial v}\right)^{2}-\left(\frac{\partial a}{\partial v}\right)^{2}=0
$$

If $\xi^{3} \not \equiv 0$, then $p_{1}=0, p_{2}=0, \partial a / \partial v=\partial b / \partial v=0$. We have a conic of fixed axes generating a right cylinder.

If $\xi^{3}=0$, we have a fixed centre, and either

$$
p_{1}=0, \quad\left(\frac{\partial b}{\partial v}\right)^{2}-\left(\frac{\partial a}{\partial v}\right)^{2}=p_{2}^{2} a^{2}
$$

or

$$
p_{2}=0, \quad\left(\frac{\partial a}{\partial v}\right)^{2}-\left(\frac{\partial b}{\partial v}\right)^{2}=p_{1}^{2} b^{2}
$$

The distance between the foci is constant, the plane rotates about one axis which has a fixed direction.

There is the second case where

$$
E=a^{2}=b^{2}, \quad F=\left[a \xi^{2} \cos u-a \xi^{1} \sin u+p_{3} a^{2}\right] .
$$

We get from $\partial\left(E G-F^{2}\right) / \partial u=0$ that

$$
\begin{gathered}
G=\left[\xi^{2} \cos u-\xi^{1} \sin u+p_{3} a\right]^{2}+\phi(v), \\
\xi^{1} \frac{\partial a}{\partial v}-\xi^{3} a p_{2}=0, \quad \xi^{2} \frac{\partial a}{\partial v}+\xi^{3} a p_{1}=0, \\
a^{2}\left(p_{1}^{2}-p_{2}^{2}\right)=\left(\xi^{1}\right)^{2}-\left(\xi^{2}\right)^{2}, \quad a^{2} p_{1} p_{2}=\xi^{1} \xi^{2} .
\end{gathered}
$$


One solution of these equations is $p_{1}=p_{2}=0, \xi^{1}=\xi^{2}=0$, and this gives rise to the parallels of a surface of revolution.

A second solution is $\partial a / \partial v=0, \xi^{3}=0, \xi^{1}= \pm a p_{1}, \xi^{2}= \pm a p_{2}$. It is readily shown that the last three of these equations constitute necessary and sufficient conditions that the centre of the moving circle lie on and move in the direction of the characteristic line of the plane of the circle and have a velocity which is $\pm a$ times the angular velocity with which this plane turns about the characteristic line. The conditions also guarantee that the planes of the circles are the osculating planes of the locus of the centres. Thus, the circles have their centres in the points of a twisted curve of constant torsion $1 / a$, lie in the osculating planes of this curve, and have the constant radius $|a|\left(^{5}\right)$.

All other solutions of the four equations are imaginary.

THEOREM 5. If the central conics of a series are geodesically parallel, but are not circles, either they are the right sections of a quadric cylinder or the centre and direction of one axis is fixed, and the distance between the foci is constant. If the conics are real circles, either they are the parallels of a surface of revolution, or they have their centres in the points of a twisted curve of constant torsion $1 / a$, lie in the osculating planes of this curve and have the constant radius $|a|^{5}$.

Let us next inquire under what circumstances the conics will be lines of curvature. A plane curve will be a line of curvature if the normals to the surface all along it make the same angle with the plane, or what comes to the same thing, the normals to the curve making a certain constant angle with the plane are normal to the surface. If $C$ be the tangent of the angle which a normal to the conic makes with the $x^{3}$ axis, the direction cosines of this normal are proportional to

$$
b \cos u, \quad a \sin u, \quad-C\left(b^{2} \cos ^{2} u+a^{2} \sin ^{2} u\right)^{1 / 2} .
$$

This will be normal to the surface, that is to say, normal to $\partial x / \partial v$ if

$$
\begin{aligned}
{\left[\xi^{1} b \cos u+\xi^{2} a \sin u\right.} & \left.+b \frac{\partial a}{\partial v} \cos ^{2} u+a \frac{\partial b}{\partial v} \sin ^{2} u-p_{3}\left(b^{2}-a^{2}\right) \cos u \sin u\right]^{2} \\
& =C^{2}\left(b^{2} \cos ^{2} u+a^{2} \sin ^{2} u\right)\left(\xi^{3}+p_{1} b \sin u-p_{2} a \cos u\right)^{2} .
\end{aligned}
$$

This is to be an identity in $u$. The left side is a perfect square, hence either the right side is, or both vanish identically. Excluding this case, the right is a perfect square if $b^{2} \cos ^{2} u+a^{2} \sin ^{2} u$ is a perfect square, and this involves $a=b$ so that we have a circle. The evolutes of a circle are points, a sphere will touch the surface all along the circle, or the surface is the envelop of a one-parameter family of spheres.

Suppose, next, that each side vanishes identically and that $C=0$. Then

$$
\xi^{1}=\xi^{2}=\frac{\partial a}{\partial v}=\frac{\partial b}{\partial v}=p_{3}\left(b^{2}-a^{2}\right)=0 .
$$

(5) This possibility was pointed out to me by Professor Graustein. 
$C=0$ gives the fact that the plane of the conic is orthogonal to the surface, $\xi^{1}=\xi^{2}=0$ the centre is fixed, or moves orthogonally to the plane, $\partial a / \partial v=\partial b / \partial v$ $=0$ that the lengths of the axes are constant. If $p_{3}=0$, then $\partial x^{1} / \partial v=\partial x^{2} / \partial v=0$ and every point moves orthogonally to the plane. If $b=a$, the surface is the envelop of spheres of constant radius, and is therefore a canal surface.

If, on the other hand, $C \neq 0$, then $\xi^{3}=p_{1}=p_{2}=0$ and $\partial x^{i} / \partial v=0$, so that there is no surface, unless $b=a$, in which case we have the circles as before.

THEOREM 6. The necessary and sufficient condition that the central conics of a non-planar series should be lines of curvature is that either they be the characteristic circles on a one-parameter family of spheres, or that they be invariable in size and shape and invariant in their planes and so generate a surface of Monge.

2. Congruences. Let us pass to two-parameter systems, or congruences. Let us call the parameters $v_{1}, v_{2}$, putting subscripts 1 or 2 to the notations of $(3),(4),(6),(7),(8)$ to indicate the variable with regard to which the differentiation has been performed. Let us look for the focal points, which we described geometrically as the points where a conic meets an infinitely near one. Analytically this means that when a certain relation has been established between $v_{1}$ and $v_{2}$ we can make $u$ such a function of these variables that the tangent to the curve traced is the same as that to the conic. This again will involve three relations

$$
\frac{\partial x^{i}}{\partial v_{1}} d v_{1}+\frac{\partial x^{i}}{\partial v_{2}} d v_{2}+\lambda \frac{\partial x^{i}}{\partial u}=0 .
$$

Setting the discriminant of these three linear homogeneous equations equal to 0 , we get a cubic expression in $\cos u, \sin u$ which will have six roots.

THEOREM 7. The central conics of a congruence will usually have six focal points where they touch six surfaces or meet certain curves.

This number is in accordance with a result of Darboux's where it is shown $\left(^{6}\right)$ that where a congruence is composed of plane curves of order $m$ the number of focal points is $m(m+1)$. When our central conics are circles, two focal points are on the circle at infinity, we usually overlook them and say that the circles of a congruence touch four surfaces.

Let us now inquire under what circumstances the conics of a congruence are orthogonal to a surface. For this purpose, $u$ must be such a function of $v_{1}$ and $v_{2}$ that

$$
E \frac{\partial u}{\partial v_{1}}+F_{1}=E \frac{\partial u}{\partial v_{2}}+F_{2}=0
$$

(') Théorie Générale des Surfaces, vol. 2, p. 4. 
The condition of in tegrability will be

$$
E\left(\frac{\partial F_{1}}{\partial v_{2}}-\frac{\partial F_{2}}{\partial v_{1}}\right)+F_{1}\left(\frac{\partial F_{2}}{\partial u}-\frac{\partial E}{\partial v_{2}}\right)+F_{2}\left(\frac{\partial E}{\partial v_{1}}-\frac{\partial F_{1}}{\partial u}\right)=0
$$

Developing this at length, we get a rather fearsome equation

$$
\begin{aligned}
& \frac{a b}{2}\left[p_{3_{2}} \frac{\partial\left(a^{2}+b^{2}\right)}{\partial v_{1}}-p_{3_{1}} \frac{\partial\left(a^{2}+b^{2}\right)}{\partial v_{2}}+2\left(\xi_{1}^{1} \xi_{2}^{2}-\xi_{2} \xi_{1}^{2}\right)\right] \\
& \quad+\cos u\left[\frac{b \xi_{2}^{2}}{2} \frac{\partial\left(a^{2}+b^{2}\right)}{\partial v_{1}}-\frac{b \xi_{1}^{2}}{2} \frac{\partial\left(a^{2}+b^{2}\right)}{\partial v_{2}}+a^{2} b\left(p_{3_{2}} \xi_{1}^{1}-p_{3_{1}} \xi_{2}\right)\right] \\
& \quad+\sin u\left[\frac{a \xi_{1}^{1}}{2} \frac{\partial\left(a^{2}+b^{2}\right)}{\partial v_{2}}-\frac{a \xi_{2}^{1}}{2} \frac{\partial\left(a^{2}+b^{2}\right)}{\partial v_{1}}+a b^{2}\left(p_{3_{2}} \xi_{1}^{2}-p_{3_{1}} \xi_{2}^{2}\right)\right] \\
& \quad+\left(b^{2} \cos ^{2} u+a^{2} \sin ^{2} u\right)\left(\frac{\partial\left(a b p_{3_{2}}\right)}{\partial v_{1}}-\frac{\partial\left(a b p_{3_{1}}\right)}{\partial v_{2}}\right. \\
& \left.\quad+\frac{\sin u \cos u}{4}\left[\frac{\partial\left(\left(b^{2}+a^{2}\right),\left(b^{2}-a^{2}\right)\right)}{\partial\left(v_{1}, v_{2}\right)}\right)\right]+\cos ^{3} u b^{2}\left(\frac{\partial b \xi_{1}^{2}}{\partial v_{2}}-\frac{\partial b \xi_{2}^{2}}{\partial v_{1}}\right) \\
& \quad+\cos { }^{2} u \sin ^{2} u\left[b^{2}\left(\frac{\partial a \xi_{2}^{1}}{\partial v_{1}}-\frac{\partial a \xi_{1}^{1}}{\partial v_{2}}\right)+\frac{a}{2}\left(\frac{\partial\left(b^{2}-a^{2}\right)}{\partial v_{2}} \xi_{1}^{1}-\frac{\partial\left(b^{2}-a^{2}\right)}{\partial v_{1}} \xi_{2}^{1}\right)\right] \\
& \quad+\cos u \sin ^{2} u\left[a^{2}\left(\frac{\partial b \xi_{1}^{2}}{\partial v_{2}}-\frac{\partial b \xi_{2}^{2}}{\partial v_{1}}\right)+\frac{b}{2}\left[\frac{\partial\left(b^{2}-a^{2}\right)}{\partial v_{2}} \xi_{1}^{2}-\frac{\partial\left(b^{2}-a^{2}\right)}{\partial v_{1}} \xi_{2}^{2}\right]\right. \\
& \left.\quad+\sin ^{3} u a^{2}\left(\frac{\partial a \xi_{2}^{1}}{\partial v_{1}}-\frac{\partial a \xi_{1}^{1}}{\partial v_{2}}\right)\right]=0 .
\end{aligned}
$$

If we replace $u$ by $t$ as before, this equation becomes sextic.

THEOREM 8. If the central conics of a congruence be normal to more than six surfaces, the congruence is a normal one.

The conditions for a normal congruence will, then, be that the left side of this equation should vanish identically, for all values of $u$. Now if we have an expression

$$
\begin{aligned}
& A_{0}+A_{1} \cos u+B_{1} \sin u+A_{2} \cos ^{2} u+B_{2} \cos u \sin u \\
& \quad+C_{2} \sin ^{2} u+A_{3} \cos ^{3} u+B_{3} \cos ^{2} u \sin u+C_{3} \cos u \sin ^{2} u+D_{3} \sin ^{3} u=0
\end{aligned}
$$

we shall find the conditions for vanishing identically by putting $u$ successively equal to $0, \pi / 4, \pi, 2,3 \pi / 4, \pi, 3 \pi / 4,3 \pi / 2,2 \pi / 4$. This will give

$$
B_{2}=A_{0}+A_{2}=A_{0}+C_{2}=A_{1}+A_{3}=B_{1}+D_{3}=A_{1}+C_{3}=B_{1}+B_{3}=0 .
$$


Assuming, then, that $b^{2} \neq a^{2}$, we find

$$
A_{0}=A_{2}=B_{2}=C_{2}=0 ; A_{1}+A_{3}=A_{1}+C_{3}=B_{1}+B_{3}=B_{1}+D_{3}=0 \text {; }
$$

$$
\begin{gathered}
\frac{\partial(a, b)}{\partial\left(v_{1}, v_{2}\right)}=0 ; \\
\frac{\partial\left(p_{3_{1}} a b\right)}{\partial v_{2}}-\frac{\partial\left(p_{3_{2}} a b\right)}{\partial v_{1}}=0 ; \\
\frac{1}{2}\left[p_{3_{2}} \frac{\partial\left(a^{2}+b^{2}\right)}{\partial v_{1}}-p_{3_{1}} \frac{\partial\left(a^{2}+b^{2}\right)}{\partial v_{2}}\right]+\xi_{1}^{1} \xi_{2}^{2}-\xi_{1}^{2} \xi_{2}^{1}=0 ; \\
\frac{\partial}{\partial v_{2}}\left(\frac{b \xi_{1}^{2}}{\left(b^{2}-a^{2}\right)^{1 / 2}}\right)=\frac{\partial}{\partial v_{1}}\left(\frac{b \xi_{2}^{2}}{\left(b^{2}-a^{2}\right)^{1 / 2}}\right) ; \\
\frac{\partial}{\partial v_{2}}\left(\frac{a \xi_{1}^{1}}{\left(b^{2}-a^{2}\right)^{1 / 2}}\right)=\frac{\partial}{\partial v_{1}}\left(\frac{a \xi_{2}^{1}}{\left(b^{2}-a^{2}\right)^{1 / 2}}\right) ; \\
\left(p_{3_{2} \xi_{1}}^{1}-p_{3_{1}} \xi_{2}^{1}\right)+\xi_{2}^{2} \frac{\partial}{\partial v_{1}} \log \frac{a^{2}}{\left(b^{2}-a^{2}\right)^{1 / 2}}-\xi_{1}^{2} \frac{\partial}{\partial v_{2}} \log \frac{a^{2}}{\left(b^{2}-a^{2}\right)^{1 / 2}}=0 ; \\
\left(p_{3_{2}} \xi_{1}^{2}-p_{3_{1}} \xi_{2}^{2}\right)-\xi_{2}^{1} \frac{\partial}{\partial v_{1}} \log \frac{b^{2}}{\left(b^{2}-a^{2}\right)^{1 / 2}}+\xi_{1}^{1} \frac{\partial}{\partial v_{2}} \log \frac{b^{2}}{\left(b^{2}-a^{2}\right)^{1 / 2}}=0 .
\end{gathered}
$$

The most important of these equations is (I) which gives

THEOREM 9. The semi-axes of the central conics of a normal congruence are functionally related.

Let us next assume that we are in the special case where

$$
\xi_{1}^{1} \xi_{2}^{2}-\xi_{1}^{2} \xi_{2}^{1}=0 \text {. }
$$

This means geometrically that either we have a fixed centre, or the centre traces a curve, or that at each centre the plane of the conic is orthogonal to the tangent plane to the surface of centres. From (III) follows

THEOREM 10. If the central conics of a normal congruence have axes of fixed lengths, the centre will be fixed, or trace a curve, or a surface orthogonal at each point to the plane of the corresponding conic.

Assuming that (16) still holds, but the lengths of both axes are not fixed, we write

$$
\begin{aligned}
& \xi^{1} d t=\xi_{1}^{1} \frac{d v_{1}}{d t} d t+\xi_{2}^{1} \frac{d v_{2}}{d t} d t=0, \\
& \xi^{2} d t=\xi_{1}^{2} \frac{d v_{1}}{d t} d t+\xi_{2}^{2} \frac{d v_{2}}{d t} d t=0,
\end{aligned}
$$




$$
\begin{aligned}
d a & =\frac{\partial a}{\partial v_{1}} \frac{d v_{1}}{d t} d t+\frac{\partial a}{\partial v_{2}} \frac{d v_{2}}{d t} d t=0, \\
p_{3} d t & =p_{3_{1}} \frac{d v_{1}}{d t} d t+p_{3_{2}} \frac{d v_{2}}{d t} d t=0 .
\end{aligned}
$$

Here $t$ is an arbitrary variable not $\tan v / 2$. By (16) the first two have a common solution, and then, by (I), (II) and (III) the last two have a common solution unless $a^{2}+b^{2}=$ const. Suppose, first, that all four have a common solution. We are then at liberty to assume

$$
\frac{\partial a}{\partial v_{2}}=\frac{\partial b}{\partial v_{2}}=p_{3_{2}}=\xi_{2}^{1}=\xi_{2}^{2}=0 .
$$

We have also the additional equations

$$
\frac{\partial \xi_{1}^{1}}{\partial v_{2}}=\frac{\partial \xi_{1}^{2}}{\partial v_{2}}=\frac{\partial p_{3_{1}}}{\partial v_{2}}=0
$$

All seven of our equations (I)-(VII) are satisfied. Assuming that we have a surface of centres, the curves $v_{1}=$ const. thereon correspond to constant values for the lengths of the axes. Let us take $v_{2}=$ const. as the curves orthogonal to them. The equations $\xi_{2}^{1}=\xi_{2}^{2}=0$ tell us that the curves $v_{1}=$ const. are orthogonal to the corresponding planes of the conics, hence the curves $v_{2}=$ const. are tangent to the planes of the conics, or lie in them. But now we find from the first two equations (7) that the instantaneous motion of every point of the conic, when $v_{1}$ is constant, is orthogonal to the plane. The equations

$$
\frac{\partial \xi_{1}^{1}}{\partial v_{2}}=\frac{\partial \xi_{1}^{2}}{\partial v_{2}}=0
$$

give by (4)

$$
\frac{\partial}{\partial v_{2}}\left(a_{k 1} \frac{\partial X_{0}^{k}}{\partial v_{1}}\right)=\frac{\partial}{\partial v_{2}}\left(a_{k 2} \frac{\partial X_{0}^{k}}{\partial v_{1}}\right)=0 .
$$

Since $\partial X_{0} / \partial v_{1}$ is in the plane of the conic

$$
\begin{aligned}
\frac{\partial X_{0}^{j}}{\partial v_{1}} & =\lambda a_{j 1}+\mu a_{j 2}=\left(a_{k 1} \frac{\partial X_{0}^{k}}{\partial v_{1}}\right) a_{j 1}+\left(a_{k 2} \frac{\partial X_{1}^{k}}{\partial v_{1}}\right) a_{j 2} \\
\frac{\partial^{2} X_{0}^{j}}{\partial v_{1} \partial v_{2}} & =\left(a_{k 1} \frac{\partial X_{0}^{k}}{\partial v_{1}}\right) \frac{\partial a_{j 1}}{\partial v_{2}}+\left(a_{k 2} \frac{\partial X_{0}^{k}}{\partial v_{1}}\right) \frac{\partial a_{j 2}}{\partial v_{2}} .
\end{aligned}
$$

But by (4) and $p_{3_{2}}=0$ 


$$
\left(a_{j 1} \frac{\partial a_{j 1}}{\partial v_{2}}\right)=\left(a_{j 1} \frac{\partial a_{j 2}}{\partial v_{2}}\right)=\left(a_{j 2} \frac{\partial a_{j 1}}{\partial v_{2}}\right)=\left(a_{j 2} \frac{\partial a_{j 2}}{\partial v_{2}}\right)=0 .
$$

Hence

$$
\left(a_{j 1} \frac{\partial^{2} X_{0}^{j}}{\partial v_{1} \partial v_{2}}\right)=\left(a_{j 2} \frac{\partial^{2} X_{0}^{j}}{\partial v_{1} \partial v_{2}}\right)=0 .
$$

Hence $\partial^{2} X_{0}^{j} / \partial v_{1} \partial v_{2}=\rho\left(\partial X_{0}^{j} / \partial v_{2}\right)$ and

$$
\frac{\partial}{\partial v_{1}}\left(\frac{\frac{\partial X_{0}^{1}}{\partial v_{2}}}{\frac{\partial X_{0}^{3}}{\partial v_{2}}}\right)=\frac{\partial}{\partial v_{1}}\left(\frac{\frac{\partial X_{0}^{2}}{\partial v_{2}}}{\frac{\partial X_{0}^{3}}{\partial v_{2}}}\right)=0 .
$$

This means that the normals to the plane all along the curve $v_{2}=$ const. are parallel and this curve must be in the plane, not merely tangent to it. There are, hence, only a singly infinite number of planes, so that in each there are an infinite number of conics. We have moreover the equations

$$
\frac{\partial \xi_{1}^{1}}{\partial v_{2}}=\frac{\partial \xi_{1}^{2}}{\partial v_{2}}=\frac{\partial p_{3_{1}}}{\partial v_{2}}=0 .
$$

From the equations ( 7 ) when $v=v_{1}$ everything is independent of $v_{2}$. Hence we have the same series of conics in all our planes. The congruence is generated by an immovable set of conics in a singly infinite set of planes. Conversely, such a set of conics will clearly generate a normal congruence.

When the centre traces a curve, if $\xi_{2}^{3} \neq 0$ we may repeat our previous reasoning, $\partial X_{0}^{i} / \partial v_{2} \not \equiv 0$ and we have in each plane a one-parameter family of concentric conics. If $\xi_{2}^{3}=0, \partial X_{0}^{i} / \partial v_{2}=0$, then

$$
\frac{\partial}{\partial v_{2}}\left(a_{k 1} \frac{\partial X_{0}^{k}}{\partial v_{1}}\right)=\frac{\partial}{\partial v_{2}}\left(a_{k 2} \frac{\partial X_{0}^{k}}{\partial v_{1}}\right)=0, \quad \frac{\partial a_{k 1}}{\partial v_{2}} \frac{\partial X_{0}^{k}}{\partial v_{1}}=\frac{\partial a_{k 2}}{\partial v_{2}} \frac{\partial X_{0}^{k}}{\partial v_{2}}=0 .
$$

As before $\partial X_{0}^{j} / \partial v_{1}=\lambda a_{j 1}+\mu a_{j 2}, \xi_{1}^{3}=0$. This means that the curve traced by the centre is tangent to the plane of the conic. Our last expression can be written better

$$
\frac{\partial X_{0}^{j}}{\partial v_{1}}=\left(a_{k 1} \frac{\partial X_{0}^{k}}{\partial v_{1}}\right) a_{j 1}+\left(a_{k 2} \frac{\partial X_{0}^{k}}{\partial v_{1}}\right) a_{j 2} .
$$

Remembering that the left is independent of $v_{2}$ and $p_{3_{2}}=0$,

$$
\frac{\partial X_{0}^{j}}{\partial v_{1}} \frac{\partial a_{j 1}}{\partial v_{1}}=\left(a_{k 1} \frac{\partial X_{0}^{k}}{\partial v_{1}}\right) p_{3_{1}} \text {. }
$$


Differentiating to $v_{2}$, remembering $\partial X_{0}^{j} / \partial v_{2}=p_{3_{2}}=\partial p_{3_{2}} / \partial v_{2}=0$,

$$
\frac{\partial X_{0}^{j}}{\partial v_{1}} \frac{\partial^{2} a_{j 1}}{\partial v_{1} \partial v_{2}}=\frac{\partial X_{0}^{j}}{\partial v_{1}} \frac{\partial^{2} a_{j 2}}{\partial v_{1} \partial v_{2}}=0
$$

Differentiating to $v_{1}$,

$$
\frac{\partial^{2} X_{0}^{j}}{\partial v_{1}^{2}} \frac{\partial a_{j 2}}{\partial v_{2}}=\frac{\partial^{2} X_{0}^{j}}{\partial v_{1}^{2}} \frac{\partial a_{j 1}}{\partial v_{2}}=0
$$

Hence

$$
A \frac{\partial^{2} X_{0}^{j}}{\partial v_{1}^{2}}+B \frac{\partial X_{0}^{j}}{\partial v_{1}}+C X_{0}^{j}=0
$$

The centre traces a plane curve through the fixed origin. As this can be anywhere, the curve must be a straight line. Hence we have a series of conics whose centres lie on a line while the plane is rotated about that line.

There remains the possibility that the centre of the conics should be fixed. Here we are back on the first case, we have a set of invariable conics whose planes envelop a cone with its vertex at their common centre. The reasoning is reversible in each case. We note that in every case we have a one-parameter family of conics immovable in a moving plane.

I return to the equations (17) and (18) and assume that the first two still have a common solution, and hence, that the last two have, when $a^{2}+b^{2}$ is not a constant, but that the solutions are different. Here we are free to assume that

$$
\frac{\partial a}{\partial v_{2}}=p_{3_{2}}=0, \quad \xi_{1}^{1}=\xi_{1}^{2}=0
$$

From (IV) and (V),

$$
\xi_{2}^{1}=\frac{\left(b^{2}-a^{2}\right)^{1 / 2}}{a} \phi_{2}\left(v_{2}\right), \quad \xi_{2}^{2}=\frac{\left(b^{2}-a^{2}\right)^{1 / 2}}{b} \psi_{2}\left(v_{2}\right) .
$$

From (II), $p_{3_{1}}$ is a function of $v_{1}$ alone. From (VI), $\xi_{2}^{2} / \xi_{2}^{1}$ is a function of $v_{1}$ alone. Hence $\psi_{2} / \phi_{2}$ is a constant. We may change variables writing $\phi_{2}=v_{2}, \psi_{2}=k v_{2}$. Then, from (VI) and (VII)

$$
\begin{aligned}
p_{3_{1}}=\frac{a}{k b} \frac{\partial}{\partial v_{1}} \log \frac{a^{2}}{\left(b^{2}-a^{2}\right)^{1 / 2}} & =-\frac{k b}{a} \frac{\partial}{\partial v_{1}} \log \frac{b^{2}}{\left(b^{2}-a^{2}\right)^{1 / 2}}, \\
p_{3_{1}}\left(k \frac{b}{a}+\frac{a}{k b}\right) & =2 \frac{\partial}{\partial v_{1}} \log \frac{a}{b} .
\end{aligned}
$$


Putting $a / b=\rho$,

$$
p_{3_{1}}=\frac{2\left(k^{2} p^{2}+1\right)}{k \rho^{2}} \frac{\partial \rho}{\partial v_{1}} .
$$

Again, eliminating $p_{3_{1}}$ from (VI) and (VII)

$$
a^{2} \frac{\partial}{\partial v_{1}} \log \frac{a^{2}}{\left(b^{2}-a^{2}\right)^{1 / 2}}+k^{2} b^{2} \frac{\partial}{\partial v_{1}} \log \frac{b^{2}}{\left(b^{2}-a^{2}\right)^{1 / 2}}=0 .
$$

This can be written

$$
\begin{aligned}
2 a \frac{\partial a}{\partial v_{1}}+2 k^{2} b^{2} \frac{\partial b}{\partial v_{1}} & =\left(a^{2}+k^{2} b^{2}\right) \frac{\partial \log \left(b^{2}-a^{2}\right)^{1 / 2}}{\partial v_{1}}, \\
a^{2}+k^{2} b^{2} & =C\left(b^{2}-a^{2}\right),
\end{aligned}
$$

where $C$ is a constant since $a$ and $b$ are not functions of $v_{2}$. Hence $\rho=$ const., $p_{3_{1}}=0$. Hence from (VI) and (VII) we either have $a$ and $b$ constant which gives $\partial a / \partial v_{1}=\partial b / \partial v_{1}=0$ and throws us back on a previous case, or else

$$
\xi_{2}^{2}=\xi_{2}^{1}=p_{3_{2}}=0 \text {. }
$$

Here the centre is either fixed or traces a curve orthogonal to the plane of the conic. Again we are on a previous case.

Next we assume $a^{2}+b^{2}=$ const. Then from (III) the equations (17) have a common solution. Assume that this is a solution of the first equation (18). We may write

$$
\xi_{2}^{1}=\xi_{2}^{2}=\frac{\partial a}{\partial v_{2}}=0
$$

From (VI) and (VII)

$$
p_{3} \xi_{1}^{1}=p_{3_{2}} \xi_{1}^{2}=0
$$

If $p_{3_{2}}=0$, we have equations (19) and (20) and we can proceed as before. If $\xi_{1}^{1}=\xi_{1}^{2}=0$, the centre is either fixed or traces a curve which cuts the plane orthogonally. There is but a singly infinite set of planes. We may take the parameter $v_{2}$ to give the plane. As $\partial a / \partial v_{2}=\partial b / \partial v_{2}=0$, we have in each plane the same set of concentric conics.

Suppose now that the solutions of (17) do not give a solution of (18).

We may write

$$
\frac{\partial a}{\partial v_{2}}=\frac{\partial b}{\partial v_{2}}=0, \quad \xi_{1}^{1}=\xi_{1}^{2}=0 .
$$

We may assume $a=\cos v_{1}, b=\sin v_{1}$. From (V) and (IV)

$$
\xi_{2}^{1}=\phi_{1}\left(v_{2}\right)\left(\tan ^{2} v_{1}-1\right)^{1 / 2}, \quad \xi_{2}^{2}=\phi_{2}\left(v_{2}\right) \frac{\left(\tan ^{2} v_{1}-1\right)^{1 / 2}}{\tan v_{1}}
$$


Now if $p_{3_{1}}=0, \partial p_{3_{2}} / \partial v_{1}=0$, and we are back on a previous case. If $p_{3_{1}} \neq 0$, we eliminate it between the equations (VI) and (VII) and find that $\xi_{2}^{1} / \xi_{2}^{2}$ is a function of $v_{1}$ alone. Hence $\phi_{2}\left(v_{2}\right)=k\left(\phi_{1}\left(v_{2}\right)\right)$ where $k$ is constant, and

$$
\begin{aligned}
\partial \log \frac{a^{2}}{\left(b^{2}-a^{2}\right)^{1 / 2}}+\left(\frac{\xi_{2}^{1}}{\xi_{1}^{2}}\right)^{2} \partial \log \frac{b^{2}}{\left(b^{2}-a^{2}\right)^{1 / 2}} & =0 \\
\frac{-\tan ^{3} v_{1}}{\tan ^{2} v_{1}-1}-\frac{\operatorname{ctn}^{3} v_{1} \tan ^{2} v_{1}}{1-\operatorname{ctn}^{2} v_{1}} k^{2} & =0 \\
\frac{\tan ^{2} v_{1}+k^{2}}{\tan ^{2} v_{1}-1} & =0
\end{aligned}
$$

so that $\tan v_{1}$ is constant and we do not have a two-parameter family.

THEOREM 11. If in a normal congruence of central conics the locus of the centres is a surface which at each point is orthogonal to the plane of the corresponding conic, or is a curve, or is fixed, the congruence is generated by a series of conics which are immovable in a moving plane.

There remains the case where the first two equations (17) are not proportional. We may assume here $\xi_{2}^{1}=\xi_{1}^{2}=0$.

Let us write

$$
\log \frac{a_{2}}{\left(b^{2}-a^{2}\right)^{1 / 2}}=A, \quad \log \frac{b^{2}}{\left(b^{2}-a^{2}\right)^{1 / 2}}=B .
$$

From (IV) and (V)

$$
\xi_{2}^{2}=\frac{\left(b^{2}-a^{2}\right)^{1 / 2}}{b} \phi_{2}\left(v_{2}\right), \quad \xi_{1}^{1}=\frac{\left(b^{2}-a^{2}\right)^{1 / 2}}{a} \phi_{1}\left(v_{1}\right) .
$$

We now change the variables $v_{1}$ and $v_{2}$ to such functions of them that

$$
\begin{aligned}
\frac{\partial w_{1}}{\partial v_{1}} & =\phi_{1}, & \frac{\partial w_{2}}{\partial v_{2}} & =\phi_{2}, \\
\phi_{1}\left(v_{1}\right) & =\psi_{1}\left(w_{1}\right), & \phi_{2}\left(v_{2}\right) & =\psi_{2}\left(w_{2}\right), \\
p_{3_{2}} & =\frac{-a \psi_{2}}{b} \frac{\partial A}{\partial w_{1}}, & p_{3_{1}} & =\frac{b \psi_{1}}{a} \frac{\partial B}{\partial w_{2}} .
\end{aligned}
$$

Further, let

From (II) and (III)

$$
a^{2} \frac{\partial A}{\partial v_{1}}=\alpha, b^{2} \frac{\partial B}{\partial w_{2}}=\beta
$$




$$
\begin{array}{r}
\frac{\partial \alpha}{\partial w_{1}}+\frac{\partial \beta}{\partial w_{2}}=0, \\
\alpha \frac{\partial\left(a^{2}+b^{2}\right)}{\partial w_{1}}+\beta \frac{\partial\left(a^{2}+b^{2}\right)}{\partial w_{2}}=0 .
\end{array}
$$

I confess, to my shame, that I have not been able to make much progress towards solving these equations, or discovering their geometrical significance. In spite of that I still think that the method here outlined is the most promising for studying the problems indicated.

HARVARD UNIVERSITY,

CAmbridge, Mass. 